\title{
A Randomized Controlled Trial of Mindfulness- Based Stress Reduction to Prevent Flare-Up in Patients with Inactive Ulcerative Colitis
}

\author{
S. Jedel ${ }^{a} \quad$ A. Hoffman ${ }^{\text {d, e }}$ P. Merriman ${ }^{f} \quad$ B. Swansong, h $\quad$ R. Voigt ${ }^{a} \quad$ K.B. Rajang \\ M. Shaikh ${ }^{a}$ H. Li ${ }^{a} \quad$ A. Keshavarzian ${ }^{a-c, i}$
}

${ }^{a}$ Division of Digestive Diseases and Nutrition, Department of Internal Medicine, Rush University Medical Center, ${ }^{b}$ Department of Pharmacology and ' Department of Physiology and Molecular Biophysics, The Graduate College, and ${ }^{d}$ Department of Internal Medicine, Rush University, ${ }^{e}$ Department of Medicine, Rosalind Franklin University, fDepartment of Anesthesiology, Rush University, 9 Department of Internal Medicine, Institute for Healthy Aging, Rush University Medical Center, and h Nursing College, Rush University, Chicago, Ill., USA; 'Division of Pharmacology, Utrecht Institute for Pharmaceutical Sciences, Faculty of Science, Utrecht University, Utrecht, The Netherlands

\section{Key Words}

Flare-up prevention - Inactive ulcerative colitis .

Inflammatory Bowel Disease Quality of Life Questionnaire .

Mindfulness-based stress reduction - Quality of life .

Ulcerative colitis

\begin{abstract}
Background/Aims: The primary therapeutic goals in ulcerative colitis (UC) are to maintain excellent quality of life (QOL) by treating flare-ups when they occur, and preventing flareups. Since stress can trigger UC flare-ups, we investigated the efficacy of mindfulness-based stress reduction (MBSR) to reduce flare-ups and improve QOL. Methods: Patients with moderately severe UC, in remission, were randomized to MBSR or time/attention control. Primary outcome was disease status. Secondary outcomes were changes in markers of inflammation and disease activity, markers of stress and psychological assessments. Results: 55 subjects were randomized. Absence of flares, time to flare and severity of flare over 1 year were similar between the two groups. However, post hoc analysis showed that MBSR decreased the proportion of participants with at least one flare-up among those
\end{abstract}

\section{KARGER}

E-Mail karger@karger.com

www.karger.com/dig with top tertile urinary cortisol and baseline perceived stress (30 vs. $70 \% ; p<0.001$ ). MBSR patients who flared demonstrated significantly lower stress at the last visit compared to flared patients in the control group $(p=0.04)$. Furthermore, MBSR prevented a drop in the Inflammatory Bowel Disease Quality of Life Questionnaire during flare $(p<0.01)$. Conclusion: MBSR did not affect the rate or severity of flare-ups in UC patients in remission. However, MBSR might be effective for those with high stress reactivity (high perceived stress and urinary cortisol) during remission. MBSR appears to improve QOL in UC patients by minimizing the negative impact of flare-ups on QOL. Further studies are needed to identify a subset of patients for whom MBSR could alter disease course.

(c) 2014 S. Karger AG, Basel

\section{Introduction}

Ulcerative colitis (UC) is a type of chronic inflammatory bowel disease (IBD) that poses a significant health problem. UC is characterized by symptomatic periods (flare-up) interspersed with asymptomatic periods (re- 
missions) [1]. Since there is no cure for UC, the primary therapeutic goal is to improve quality of life (QOL) by preventing and promptly treating flare-ups when they occur [1]. The current management approach is daily medication during periods of remission to decrease the likelihood of flare-up [2]; however, this approach is not universally effective because: (a) medication compliance is often suboptimal $[2,3]$ and (b) at least $30-40 \%$ of patients who are medication-compliant still develop flare-ups with compromised QOL [1]. Thus, an alternative therapeutic approach to prevent flare-up is highly desirable. There is evidence that psychosocial stressors can trigger UC flare-up [4, 5], UC patients have exaggerated responses to stressors [6,7], and psychological stressors can initiate mucosal and systemic inflammatory cascades [7]. Thus, stress management approaches have the potential to prevent disease flare-ups and improve the QOL for UC patients.

Mindfulness-based stress reduction (MBSR), a widely used curriculum for teaching mindfulness [8], was originally designed as a complement to standard medical treatment and as an approach to cope with stress, by turning toward and 'facing' pain and chronic illness with self-compassion, and without judging oneself or another [8]. MBSR has been shown to: (1) reduce anxiety and distress among non-patient populations [9]; (2) reduce depression, anxiety, physical symptoms and sleep disturbance in cancer patients [10] and organ transplant patients [11], and (3) provide long-term psychological and physical benefits among people with medical conditions, such as fibromyalgia [12] and chronic pain [13]. MBSR also modulates pro-inflammatory cytokine profiles to an anti-inflammatory pattern in patients with breast or prostate cancer [14] and HIV [15], suggesting the physiological basis by which MBSR may improve inflammatory diseases. Limited data also suggest that MBSR may be effective in patients with gastrointestinal disorders. Patients with irritable bowel syndrome who participated in MBSR demonstrated significant improvement in symptom severity, QOL and psychosocial distress, compared to controls [16] and we have recently shown that state of mindfulness, in the absence of formal training, is significantly and inversely correlated with anxiety, depression and perceived stress and positively correlated with QOL in patients with inactive UC [17]. However, MBSR has not been empirically tested in patients with UC.

The aim of our randomized time/attention controlled study was to investigate the effects of MBSR on disease course, QOL, markers of inflammation, and psychologi- cal parameters in UC patients who were in remission. We hypothesized that participation in MBSR would prevent subclinical mucosal inflammation and clinical flare-up and improve QOL in UC patients.

\section{Materials and Methods}

\section{Study Participants}

Patients with inactive UC (in remission), enrolled from January 2008 through October 2010, were recruited from the Rush University Medical Center (RUMC) IBD Clinic and the Greater Chicago area. The clinical coordinator screened prospective subjects' medical records to determine eligibility.

Inclusion criteria: (1) documented moderately severe UC (Mayo UC disease activity index (Mayo UC-DAI): 6-12); (2) inactive UC at time of recruitment (Mayo UC-DAI: $<2$, sigmoidoscopy score: $0 / 1$, bleeding score: $0 / 1) ;(3)$ at least one documented disease flare-up within the past 6 months; (4) colonic involvement of $>15$ $\mathrm{cm}$ from the anal verge; (5) age 18-70; (6) taking no IBD medication or on a stable dose (5-ASA products: mesalamine, sulfasalazine or Colazal) for at least 3 weeks prior to enrollment; immunosuppressive medication (azathioprine or 6-mercaptopurine), biologics (infliximab, adalimumab or natalizumab) for at least 3 months, or prednisone $(<5 \mathrm{mg})$. If patients were taking medication, they were encouraged to stay on the current dose over the course of the study, and any changes were recorded, and (7) willingness to participate in one of two 8-week courses. To confirm inactive disease, all subjects were examined and sigmoidoscopy was performed by a gastroenterologist (A.K.). A clinical psychologist (S.J. or P.M.) interviewed patients to screen for psychiatric eligibility.

Exclusion criteria: (1) history of colon resection; (2) use of antibiotics within the previous 30 days; (3) use of antidiarrheal medications within the previous 7 days of enrollment; (4) use of nonallowed medication including prednisone $>5 \mathrm{mg} /$ day; (5) unresolved history of physical or sexual abuse ${ }^{1}$, current or past dissociative disorder, post-traumatic stress disorder ${ }^{1}$, history of psychosis, or prior hospitalization for self-harm/suicidal ideation; (6) resistance to mind/body interventions due to religious or moral beliefs; (7) current pregnancy/lactation; (8) prior mind/body intervention training; (9) current chronic disorders, such as severe cardiac, renal disease (creatinine greater than twice normal), pulmonary disease, active infection, liver disease (ALT or AST greater than twice normal) or other organ system disease/morbidity requiring medical visits $>3$ times/year and creating excess perceived stress per the determination of the psychologist.

One additional secondary endpoint that is not addressed in the current paper was to assess the impact of stress management on stool microbiota; thus, subjects could not use antibiotics within the previous 30 days. We plan to analyze the impact of MBSR on stool microbiota in the future.

1 At the time we began our study, there were no data regarding the efficacy of MBSR among individuals with PTSD or abuse, and therefore there were theoretical concerns about MBSR safety in these populations. 
Table 1. Subject demographics (MBSR vs. control)

\begin{tabular}{|c|c|c|c|}
\hline Demographic & $\begin{array}{l}\text { MBSR group } \\
(\mathrm{n}=27)\end{array}$ & $\begin{array}{l}\text { Control } \\
\text { group } \\
(n=28)\end{array}$ & $\begin{array}{l}\mathrm{p} \\
\text { value }\end{array}$ \\
\hline Age & $46.04 \pm 12.80$ & $39.68 \pm 11.06$ & $0.05^{*}$ \\
\hline \multicolumn{4}{|l|}{ Gender } \\
\hline Male & $15(55.6)$ & $9(32.1)$ & \multirow[t]{2}{*}{0.14} \\
\hline Female & $12(44.4)$ & $19(67.9)$ & \\
\hline \multicolumn{4}{|l|}{ Ethnicity/race } \\
\hline Asian & $1(3.7)$ & $1(3.6)$ & \multirow[t]{6}{*}{0.08} \\
\hline Black or African- & & & \\
\hline American & $0(0)$ & $5(17.9)$ & \\
\hline White & $26(96.3)$ & $22(78.6)$ & \\
\hline Hispanic & $3(11.1)$ & $2(7.1)$ & \\
\hline Not Hispanic & $24(88.9)$ & $26(92.9)$ & \\
\hline \multicolumn{4}{|l|}{ Marital status } \\
\hline Married & $17(63.0)$ & $12(42.9)$ & \multirow[t]{4}{*}{0.30} \\
\hline Single & $8(29.6)$ & $14(50.0)$ & \\
\hline Divorced or legally & & & \\
\hline separated & $2(7.4)$ & $2(7.1)$ & \\
\hline \multicolumn{4}{|l|}{ Work status ${ }^{1}$} \\
\hline Full-time & $21(77.8)$ & $19(64.3)$ & \multirow[t]{5}{*}{$0.05^{*}$} \\
\hline Part-time & $2(7.4)$ & $3(10.7)$ & \\
\hline $\begin{array}{c}\text { Unemployed, retired, } \\
\text { or homemaker }\end{array}$ & $2(7.4)$ & $3(10.7)$ & \\
\hline Student & $0(0)$ & $1(3.4)$ & \\
\hline Disability & $0(0)$ & $1(3.4)$ & \\
\hline Age at diagnosis & $38.72 \pm 13.55$ & $31.48 \pm 9.82$ & $0.03^{*}$ \\
\hline \multicolumn{4}{|l|}{ Extent of disease ${ }^{2}$} \\
\hline Left-sided & $12(44.4)$ & $8(28.6)$ & \\
\hline Pancolitis & $14(51.9)$ & $18(64.3)$ & \\
\hline Total number of flare-ups & $4.3 \pm 2.5$ & $5.1 \pm 3.5$ & 0.40 \\
\hline Flare-ups in the past year & $3.1 \pm 4.3$ & $1.8 \pm 0.9$ & 0.20 \\
\hline \multicolumn{4}{|l|}{ Prednisone-dependent } \\
\hline Yes & $11(40.7)$ & $13(46.4)$ & \multirow[t]{2}{*}{0.70} \\
\hline No & $16(59.3)$ & $15(53.6)$ & \\
\hline \multicolumn{4}{|c|}{ Current IBD medications $^{3}$} \\
\hline None & $2(7.4)$ & $2(7.1)$ & \multirow[t]{5}{*}{0.80} \\
\hline 5-ASA & $22(81.5)$ & $22(78.6)$ & \\
\hline Immunomodulators & $7(25.9)$ & $6(21.4)$ & \\
\hline Biologic & $4(14.8)$ & $3(10.7)$ & \\
\hline Prednisone $(<5 \mathrm{mg})$ & $4(14.8)$ & $3(10.7)$ & \\
\hline \multicolumn{4}{|l|}{ Past prednisone ${ }^{4}$} \\
\hline Yes & $18(66.7)$ & $19(67.9)$ & \multirow[t]{2}{*}{0.90} \\
\hline No & $7(25.9)$ & $7(25.0)$ & \\
\hline
\end{tabular}

Values expressed as mean \pm SD. Values in parentheses are percentage. ${ }^{*} \mathrm{p}<0.05 .{ }^{1}$ Three patients did not identify work status. ${ }^{2}$ Extent of disease is unknown for 3 patients ( 1 in MBSR and 2 in control). ${ }^{3}$ Many patients were taking more than one medication, thus totals exceed $100 \%$.

\section{Study Design}

This was a randomized (concealed), double-blind, time/attention controlled study. Patients were informed that they would be randomized to one of two mind/body courses, both of which had been shown to benefit other aspects of health and well-being and had never been tested in UC. During the first study visit, baseline data were collected (i.e. self-report questionnaires, blood draw, and 24-hour urine and stool collection) and subjects were assigned to either the MBSR or time/attention control group based on a computer-generated randomized block design that was supplied by the trial statistician (H.L.). A block randomization of size 4 and 6 were used to randomly assign patients. The randomization allocation (ratio of 1:1) was in a sealed envelope and the clinical coordinator did not have knowledge of which arm of the study the subject would be assigned to until the envelope was opened. The primary investigators and staff involved with assessments were blinded until completion of the study, analysis of the collected data, and submission of reports to the Data Safety Monitoring Board. The statistician was also blinded, with the exception of having access to a subject's identification number that links patient data to group characteristics for analysis purposes. Both courses began within 1 month of the baseline study visit. Patients were blinded to the study hypothesis (MBSR is effective in preventing UC flare-up), had no knowledge of the content of either course before randomization and had no knowledge of the content of the course that they were not assigned to. The courses occurred on different parts of the RUMC hospital campus, to control for dissemination of treatment effects. Of course, the instructors were not blinded to the class they were teaching (MBSR or control), but their knowledge had nothing to do with trial outcomes as they had no influence in assessing outcomes. The investigator (A.K.) who assessed the patients for outcomes and the readouts for primary and secondary outcomes was blinded to treatment assignment.

To ensure that the gastroenterologist (A.K.) who examined the patients and assessed disease activity was blinded to group assignment throughout the study period, (1) A.K. instructed the team not to inform him which day of the week each class was held in the event that he run into a subject in the hospital on the day of his/her course, (2) A.K. instructed subjects not to give him any information about the content of the class, (3) A.K./subject interactions were witnessed by the clinical coordinator to ensure that blindness remained intact. A.K. was asked to predict the group assignment of subjects at each study visit and his response was consistently 'I do not know'. No changes to study design or definition of outcomes were made after the trial commenced. Data analysis was initiated only after all 53 study subjects had completed the study. Demographic characteristics of enrolled subjects are provided in table 1.

\section{Sample Size Justification}

The goal of this study was to determine the effect sizes of MBSR on IBD and stress-related variables. Because there are no reports of studies on MBSR efficacy in preventing or treating IBD, ours is an exploratory study designed to look at effect sizes and typical rates of flare-up in this group of patients. We will have $80 \%$ power to detect $10 \%$ difference in a smaller proportion of subjects who flared in MBSR (45 vs. 55\%) compared to the control group using 
a sample size of 412 subjects in each group with a type I error rate of $5 \%$. For one of our secondary outcomes, calprotectin, an independent $t$ test (MBSR vs. control) would provide $80 \%$ power to detect a moderate effect size of 0.23 that translates into a difference of 7 units with a common standard deviation of 31 using 315 patients in each group. Thus, based on these calculations, our study was underpowered; however, enrolling at least 730 subjects is impractical for a single-center study and is not appropriate for a phase 2, proof of principle study. Furthermore, the clinical relevance and cost/effectiveness of this potential small effect of MBSR is highly questionable.

\section{Intervention}

\section{Mindfulness-Based Stress Reduction}

The MBSR intervention followed the 8-week curriculum developed by Kabat-Zinn [18] and weekly sessions that spanned 2$2.5 \mathrm{~h}$ involved instruction and practice in formal 'exercises' or 'meditations' (i.e. sitting meditation, body scans, and yoga postures) and informal practices (awareness of personal reactions to everyday events) aimed to promote mindfulness in daily life. Homework assignments, intended to reinforce techniques and general strategies, involved $45 \mathrm{~min} /$ day of MBSR, 6 days/week, guided by compact disc. A physician (A.H.) with more than 15 years of MBSR expertise taught the course, except for two classes when a psychologist experienced in MBSR substituted.

\section{Time/Attention Control}

This intervention was used to control for the time, support and attention that subjects received in the MBSR group. The course had an identical group format to MBSR, although each weekly class was slightly shorter in duration with homework comprised of articles that supplemented the course content. The focus of the course was mind/body medicine and was comprised of six lectures and two videos. Course topics included the effect of stress on physical and psychological health, the physiology of the 'flight-or-fight response', the effect of stress on sleep, and stress and special populations (i.e. immigrants, elderly). The course was developed in conjunction with a consultant with expertise in MBSR and whose research has included a similar control group format. The course did not include any information on UC and did not teach skills for coping with stress. A clinical psychologist (S.J.) taught the weekly course.

\section{Assessments}

Biological and psychological assessments were performed at baseline (visit 1, pretreatment visit), post 8-week course (visit 2, weeks 9-12) and at 6- and 12-month follow-ups (visits 3 and 4) at RUMC. If the subject flared-up at any point during the 12-month study period, she/he completed the assessments (identical assessments to visit 4) and was removed from the study. Primary and secondary outcome measures are described below.

\section{Primary Outcome: Disease Status}

Disease status was defined as Mayo UC-DAI $>2$ plus a rectal bleeding score $>2$ and sigmoidoscopy score of $>2$. The Mayo UCDAI [19] is a commonly employed composite measure of UC disease activity that accounts for stool consistency, rectal bleeding, findings on endoscopy and physician's global assessment.

Mindfulness-Based Stress Reduction to

Prevent Flare-Up in Inactive UC

\section{Secondary Outcomes}

(a) Markers of inflammation and UC disease activity:

(i) The stool marker calprotectin was measured using the Hycult Biotech kit (catalog No. HK325). Frequency of subclinical flare-up is defined by calprotectin $>50 \mu \mathrm{g} / \mathrm{g}$ of stool.

(ii) Serum markers included: cytokines [interleukin (IL)-6, IL8 , and IL-10] and C-reactive protein (CRP). Cytokines were measured by ELISA [IL-10 (Pierce, EHIL-10), sensitivity $<3 \mathrm{pg} / \mathrm{ml}$, assay range $15.4-600 \mathrm{pg} / \mathrm{ml}$; IL-6 (Pierce, EH2IL-6), sensitivity $<1 \mathrm{pg} / \mathrm{ml}$, assay range $10.2-400 \mathrm{pg} / \mathrm{ml}$; IL-8 (Pierce, EH2IL-8), sensitivity $<2 \mathrm{pg} / \mathrm{ml}$, assay range $25.6-1,000 \mathrm{pg} / \mathrm{ml}$, and CRP (RUMC, Clinical Core Laboratory), assay range $0-8 \mu \mathrm{g} / \mathrm{ml}]$.

(iii) The Inflammatory Bowel Disease Quality of Life Questionnaire (IBD-Q) [20] is a disease-specific questionnaire used to assess health-related QOL in IBD patients. The questionnaire has four subscales: bowel symptoms, systemic symptoms, emotional factors and social factors. Items are scored on a 7-point Likert scale ranging from 1 (worst of health) to 7 (best of health) for a variety of symptoms and concerns. Scores range from 32 to 224, with higher scores representing better health.

(iv) Time to flare-up: calculated by number of days from baseline to flare-up.

(v) Severity of flare-up: defined by the UC-DAI.

(b) Markers of stress:

(i) Serum ACTH was measured after an overnight fast from blood samples obtained via intravenous catheter using an MD Bioproducts ELISA (MD Bioproducts, Division of MD Biosciences, Inc., St. Paul, Minn., USA, catalog No. M046006). Urinary cortisol was measured from urine collected over a 24-hour period prior to each study visit (R\&D Systems, Inc., Minneapolis, Minn., USA, catalog No. KGE008).

(ii) The Perceived Stress Questionnaire (PSQ) [21] was used as a validated measure of stress that assesses recent (past month) stress.

(c) Psychological assessment:

(i) The Beck Depression Inventory (BDI) [22] is an established self-report tool for screening depression and includes 21 items, each comprising 4 self-descriptive statements, which the subject rates on a 4-point scale of severity. Interpretation is based on a 0-63 total score. Test-retest reliability is $>0.90$ [23]; Spearman-Brown reliability is 0.93 , and internal consistency for test items is 0.86 [23].

(ii) The State-Trait Anxiety Inventory (STAI) [24] is a widely used self-report measure with two 20 -item scales that measure selfreported state, or current, anxiety (State-A) and trait, or chronic, anxiety (Trait-A). Individuals are asked to rate items on a 4-point scale as to how well they describe the subject's current or typical mood, from 'not at all' to 'very much so'. Scores range from 20 to 80 on each scale, with higher scores indicating greater anxiety. In the current study, only trait anxiety was measured.

(iii) The Mindful Attention Awareness Scale (MAAS) [25] measures the frequency with which individuals experience mindful states. Higher scores indicate greater frequency of mindfulness. Exploratory and confirmatory factor analyses have yielded a singlefactor model. Cronbach's $\alpha$ in an adult population was 0.87 , while 4 -week test-retest reliability was 0.81 [25]. Construct validity was supported by a significant positive correlation $(r=0.39)$ with the Mindfulness/Mindlessness Scale [26], and negative correlations with a measure of rumination ( $\mathrm{r}$ range -0.29 to -0.39 ) [25].

(iv) The Perceived Health Competence Scale (PHCS) [27] is a domain-specific, validated scale that examines the degree to which 


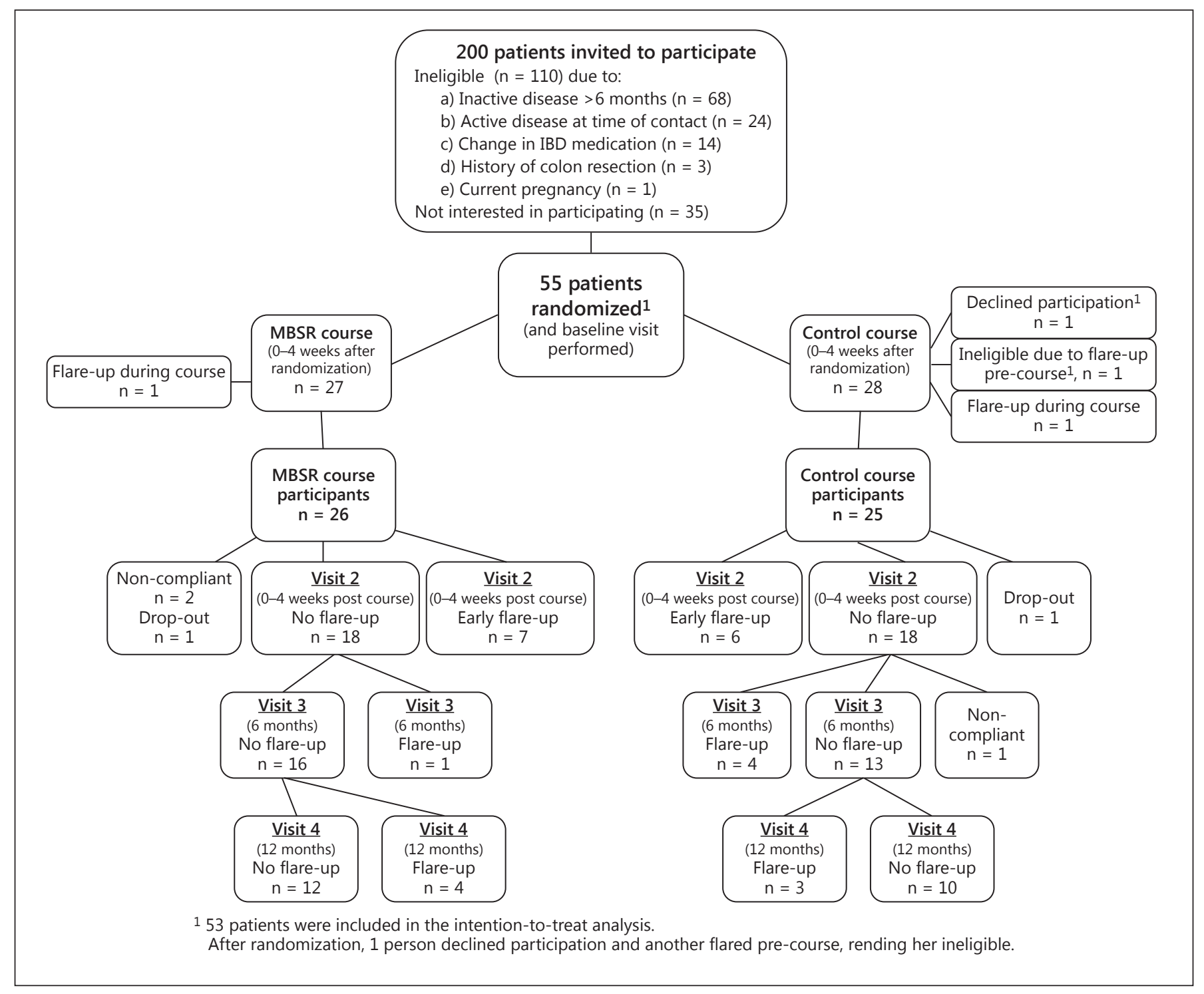

Fig. 1. Flow of randomized subjects in MBSR and control groups.

an individual perceives his ability to effectively manage health outcomes. Eight questions (5-point Likert scale) range from strongly disagree to strongly agree.

\section{Ethical Considerations}

The study was approved by the RUMC Institution Review Board and was registered at Clinicaltrials.gov (ID: NCT00568256). Patients provided written consent and received both a verbal explanation and written information regarding the study. Patients were compensated USD 300 for participation. An independent Data Safety Monitoring Board met at regular intervals throughout the study, to ensure that there were no adverse events.

\section{Statistical Analysis}

SAS version 9.2 (SAS Institute, Inc., Cary, N.C., USA) was used for all analyses. An intent-to-treat analysis was used to test for group differences in outcomes over time. Participants who flared during the intervention were included in the analysis. Student's t test and $\chi^{2}$ test were used to evaluate group equivalence at baseline. To explore the effect of treatment on psychological and clinical outcomes, a mixed random effect model (MRM) and generalized estimating equation (GEE) were performed for continuous outcomes and ordinal outcomes, respectively. The within-subject correlation of repeated measures was taken into account by a random intercept model in MRM and by assuming an exchangeable correlation structure in GEE. The ordinal outcome of sigmoidoscopy finding was modeled with a cumulative logit link in GEE model. The mod- 
Table 2. Baseline and last-visit disease and psychological characteristics (MBSR vs. control)

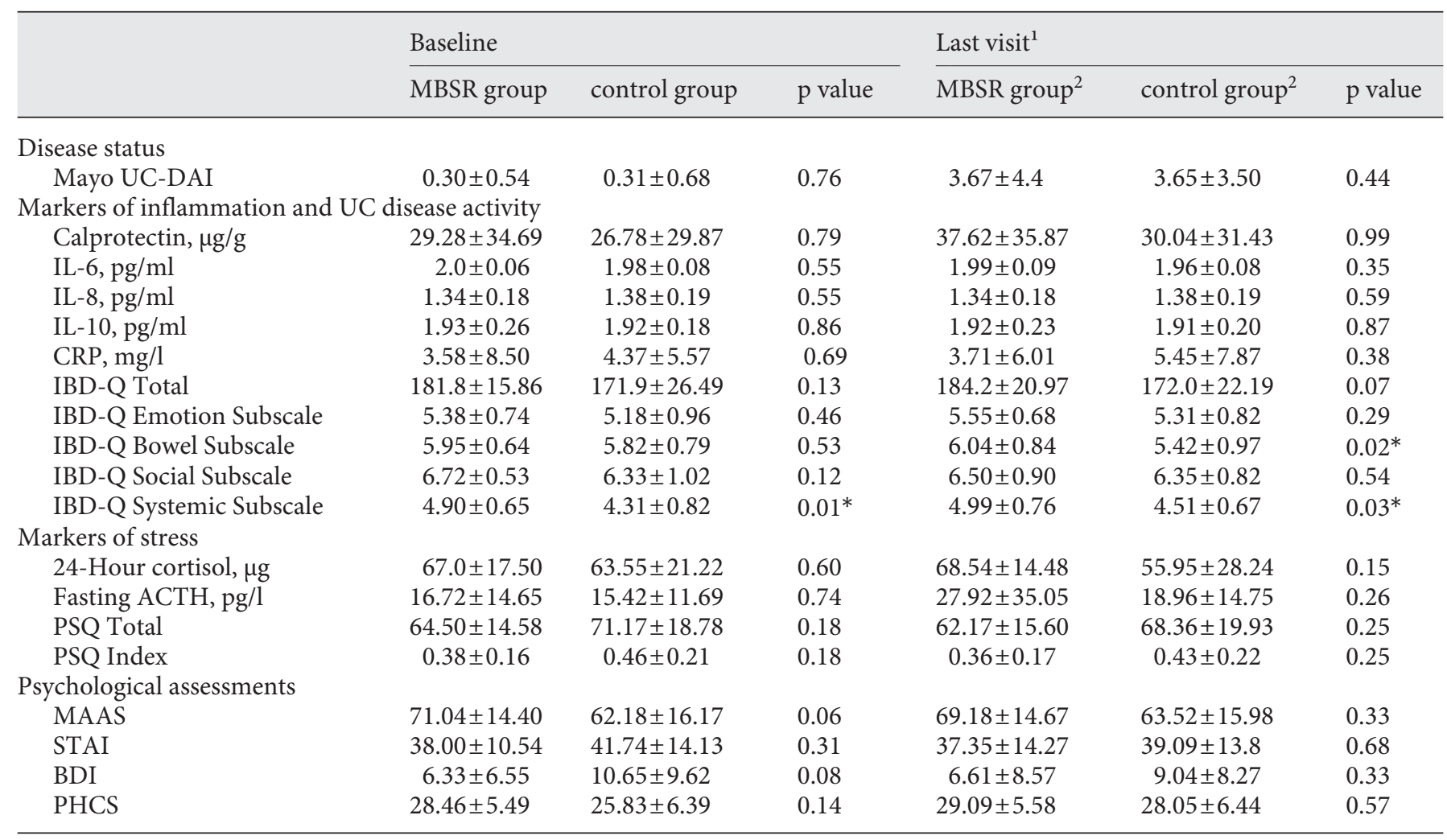

Values are expressed as mean $\pm \mathrm{SD} .{ }^{*} \mathrm{p}<0.05$

${ }^{1}$ Either 1-year follow-up or flare-up visit. ${ }^{2}$ Includes both flared and non-flared subjects.

els included coefficients for time, group, flare-up status, two-way interactions of group and time, flare-up status and time, and flareup status and group, and three-way interaction of flare-up status, group and time. In additional analyses, the MAAS and UCDAI scores were adjusted separately in the model. Comparison of the psychological and clinical markers between two treatment groups was also made for the last visit. A Kaplan-Meier survival analysis was used to test the statistical significance between the flare-up survival curves (time to flare-up) between the MBSR group and control group. Model assumptions were examined graphically and analytically and were shown to be adequately met. All multiple tests were adjusted using Bonferroni's correction for $\mathrm{p}$ values.

\section{Participant Flow during Recruitment and during the Study}

We contacted and invited 200 patients with UC to enroll in the study (fig. 1). 145 patients did not fulfill the inclusion criteria and did not qualify for the study. The most common reason was inactive disease longer than 6 months. A total of 55 patients were enrolled and randomized. Prior to the start of the course, 1 patient (randomized to the control group) declined participation and 1 patient (randomized to the control group) flared before the start of the study and therefore did not participate. A total of 53 patients (27 in MBSR and 26 in control group) were used for our intentionto-treat analysis. Two patients, 1 in each group, dropped out of the study during their 8-week course.

\section{Results}

\section{Distribution of Characteristics after Randomization}

Tables on demographic (table 1) and disease and psychological (table 2) subject characteristics after randomization show that participants in the MBSR group were an average of 7 years older than in the control group at the time of UC diagnosis and had less severe symptoms assessed by the IBD-Q Systemic Subscale. Participants in the MBSR group were also significantly older at the time of participation in the study compared to those in the control group. Stool calprotectin levels and CRP were similar at baseline between the two groups (table 2). $\mathrm{Pa}$ tients in both groups had moderately severe UC, with 
Table 3. Longitudinal analysis (baseline vs. last visit)

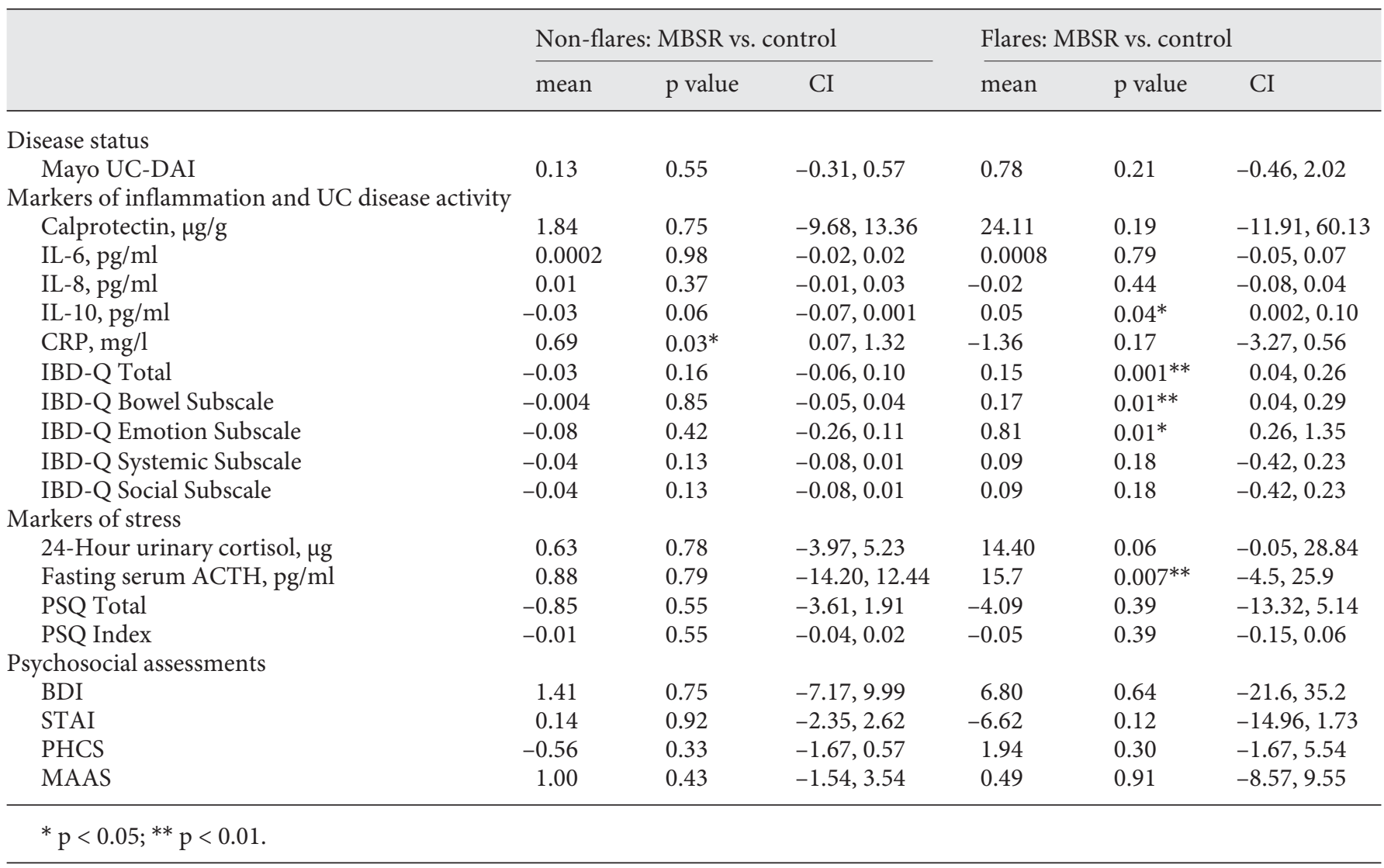

over $40 \%$ being prednisone-dependent and averaging two flare-ups during the year prior to enrollment (table 1). Course compliance was defined as participation in a minimum of 5 of 8 classes. Two patients randomized to MBSR and 1 patient randomized to the control group were not course compliant.

\section{Effect of MBSR on Outcomes}

Results for primary and secondary outcomes can be found in tables 2-4.

\section{Primary Outcome}

By the completion of the study, a total of 13 MBSR subjects $(13 / 27=48 \%)$ had flared, compared to 14 subjects in the control group $(14 / 26=54 \%)($ table $4 b)$; thus, there was not a statistically significant difference in the proportion of participants who experienced a flare-up in the year after treatment between the MBSR and time/attention control groups.

\section{Secondary Outcomes}

(a) Markers of inflammation and UC disease activity:

(i) Stool calprotectin: There were no differences in stool calprotectin between the MBSR and control groups at the time of flare-up (table 3) or in number of flare-up patients with elevated stool calprotectin values (i.e. $>50$ $\mu \mathrm{g} / \mathrm{g}$ ) at the last visit (table $4 \mathrm{~b}, \mathrm{MBSR}=7$ vs. control $=5$ ). Likewise, there were no differences between MBSR and control groups in last-visit calprotectin levels, in those who did not flare (table 4a).

(ii) Serum cytokines: Mixed model analysis showed that while serum level of anti-inflammatory cytokine IL10 increased in patients in the MBSR group who flared, the serum IL-10 decreased in those in control group who flared (table 3). However, the biological significance of this finding is not clear since proportion of participants who experienced a flare-up and the severity of flare-ups were similar between groups. There were no differences in IL-6 and IL- 8 levels between MBSR and control groups in either those who flared or those who did not (tables 3, 4). 
Table 4. Baseline and visit 4 (last visit) for non-flare subjects (a) and baseline and last visit for flare subjects (b)

\begin{tabular}{|c|c|c|c|c|c|c|c|}
\hline \multirow[t]{2}{*}{ a } & \multicolumn{3}{|l|}{ Baseline (BL) } & \multicolumn{3}{|l|}{ Visit 4 (V4) } & \multirow{2}{*}{$\begin{array}{l}\text { V4 MBSR } \\
\text { vs. V4 } \\
\text { control, } \\
\text { p value }\end{array}$} \\
\hline & $\begin{array}{l}\text { non-flare } \\
\text { MBSR } \\
(\mathrm{n}=14)\end{array}$ & $\begin{array}{l}\text { non-flare } \\
\text { control } \\
(\mathrm{n}=12)\end{array}$ & $\begin{array}{l}\text { non-flare } \\
\text { (MBSR + } \\
\text { control) } \\
(\mathrm{n}=27)\end{array}$ & $\begin{array}{l}\text { non-flare } \\
\text { MBSR }\end{array}$ & $\begin{array}{l}\text { non-flare } \\
\text { control }\end{array}$ & $\begin{array}{l}\text { non-flare } \\
\text { (MBSR + } \\
\text { control) }\end{array}$ & \\
\hline \multicolumn{8}{|l|}{ Disease status } \\
\hline $\begin{array}{l}\text { Calprotectin, } \mu \mathrm{g} / \mathrm{g} \\
\text { Subjects w/o }\end{array}$ & $23.21 \pm 35.84$ & $17.23 \pm 26.40$ & $20.49 \pm 31.32$ & $17.41 \pm 30.34$ & $12.22 \pm 18.02$ & $15.05 \pm 25.07$ & 0.63 \\
\hline calprotectin $>50 \mu \mathrm{g} / \mathrm{g}$ & $2 / 14,14.3 \%$ & $2 / 13,15.4 \%$ & $4 / 27,14.8 \%$ & $2 / 14,14.3 \%$ & $1 / 13,7.7 \%$ & $3 / 27,11.1 \%$ & 0.43 \\
\hline IL-6, pg/ml & $1.96 \pm 0.07$ & $2.00 \pm 0.09$ & $1.98 \pm 0.80$ & $1.98 \pm 0.09$ & $1.98 \pm 0.06$ & $1.98 \pm 0.07$ & 0.92 \\
\hline IL-8, pg/ml & $1.41 \pm 0.23$ & $1.50 \pm 0.19$ & $1.46 \pm 0.21$ & $1.41 \pm 0.22$ & $1.48 \pm 0.18$ & $1.45 \pm 0.20$ & 0.57 \\
\hline IL-10, pg/ml & $2.01 \pm 0.45$ & $1.92 \pm 0.15$ & $1.96 \pm 0.31$ & $1.99 \pm 0.40$ & $1.96 \pm 0.31$ & $1.97 \pm 0.34$ & 0.91 \\
\hline IBD-Q Systemic Subscale & $4.71 \pm 0.51$ & $4.30 \pm 0.91$ & $4.54 \pm 0.71$ & $4.80 \pm 0.95$ & $4.60 \pm 0.58$ & $4.71 \pm 0.79$ & 0.57 \\
\hline IBD-Q Social Subscale & $6.65 \pm 0.72$ & $6.45 \pm 1.18$ & $6.57 \pm 0.92$ & $6.64 \pm 0.83$ & $6.51 \pm 0.58$ & $6.58 \pm 0.71$ & 0.70 \\
\hline \multicolumn{8}{|l|}{ Markers of stress } \\
\hline Cortisol, $\mu \mathrm{g}$ & $63.17 \pm 16.70$ & $55.29 \pm 15.81$ & $58.92 \pm 16.06$ & $60.20 \pm 19.72$ & $53.86 \pm 27.03$ & $56.50 \pm 23.46$ & 0.65 \\
\hline $\begin{array}{l}\text { Fasting serum ACTH, } \\
\mathrm{pg} / \mathrm{ml}\end{array}$ & & & & & & & \\
\hline PSQ Total & $68.27 \pm 15.19$ & $\begin{array}{l}15.02 \pm 9.01 \\
71.33 \pm 19.31\end{array}$ & $\begin{array}{l}19.29 \pm 14.00 \\
69.65 \pm 16.76\end{array}$ & $\begin{array}{l}25.09 \pm 14.15 \\
68.73 \pm 19.03\end{array}$ & $\begin{array}{l}21.00 \pm 11.53 \\
65.88 \pm 19.24\end{array}$ & $\begin{array}{l}23.44 \pm 12.83 \\
67.53 \pm 18.63\end{array}$ & $\begin{array}{l}0.38 \\
0.75\end{array}$ \\
\hline PSQ Index & $0.43 \pm 0.17$ & $0.46 \pm 0.21$ & $0.44 \pm 0.19$ & $0.43 \pm 0.21$ & $0.40 \pm 0.21$ & $0.42 \pm 0.21$ & 0.75 \\
\hline \multicolumn{8}{|l|}{ Psychosocial assessments } \\
\hline MAAS & $73.0 \pm 13.79$ & $60.25 \pm 17.56$ & $67.63 \pm 16.35$ & $71.45 \pm 15.72$ & $62.33 \pm 16.73$ & $67.35 \pm 16.42$ & 0.23 \\
\hline BDI & $5.82 \pm 4.42$ & $11.44 \pm 9.58$ & $8.35 \pm 7.56$ & $8.64 \pm 11.07$ & $9.67 \pm 6.46$ & $9.10 \pm 9.07$ & 0.80 \\
\hline
\end{tabular}

Values expressed as mean $\pm \mathrm{SD}$.

CRP: There was a significant difference in last-visit CRP levels among flared subjects in MBSR (3.16 \pm 5.25$)$ compared to controls (table 4b, $9.09 \pm 9.01 ; \mathrm{p}=0.05$ ). There was also a difference in change in CRP levels over the 12-month time period, between MBSR and control groups among subjects who did not flare $(\mathrm{p}=0.03$; table 3). The biological significance of these effects of MBSR is doubtful.

(iii) IBD-Q: IBD-Q scores remained satisfactory (i.e. $>150$ ) in those who remained in remission regardless of randomization (tables 3, 4a). In contrast, IBD-Q scores dropped at the time of flare-up. Data from the mixed model analysis (table 3 ) demonstrated significantly better QOL among flared subjects in the MBSR group, compared to flared subjects in the control group, as measured by the IBD-Q Total Scale $(\mathrm{p}=0.001)$ and the bowel $(\mathrm{p}=0.01)$ and emotion ( $p=0.01)$ subscales, respectively. When the IBDQ Systemic Subscale at baseline was compared to the score at the time of flare-up, the score for the IBD-Q Systemic Subscale was significantly $(p=0.008)$ better in the MBSR group compared to control group (table 4b). Additionally, when comparing MBSR to the control group, and without separating flared from non-flared subjects, the MBSR group demonstrated significantly better scores on the IBD-Q Bowel Subscale ( $\mathrm{p}=0.02)$ and the IBD-Q Systemic Subscale $(\mathrm{p}=0.03)$ at last visit (table 2$)$.

(iv) Time to flare: Indeed, flare-free survival over time was similar for the MBSR and the control groups (fig. 2). Likewise, there was not a significant difference in the incidence of early flare-up (within first 3 months) between the two groups (MBSR: $7 / 27=25.9 \%$ vs. control: $6 / 26=$ 23.1\%) (fig. 1). 
Table 4 (continued)

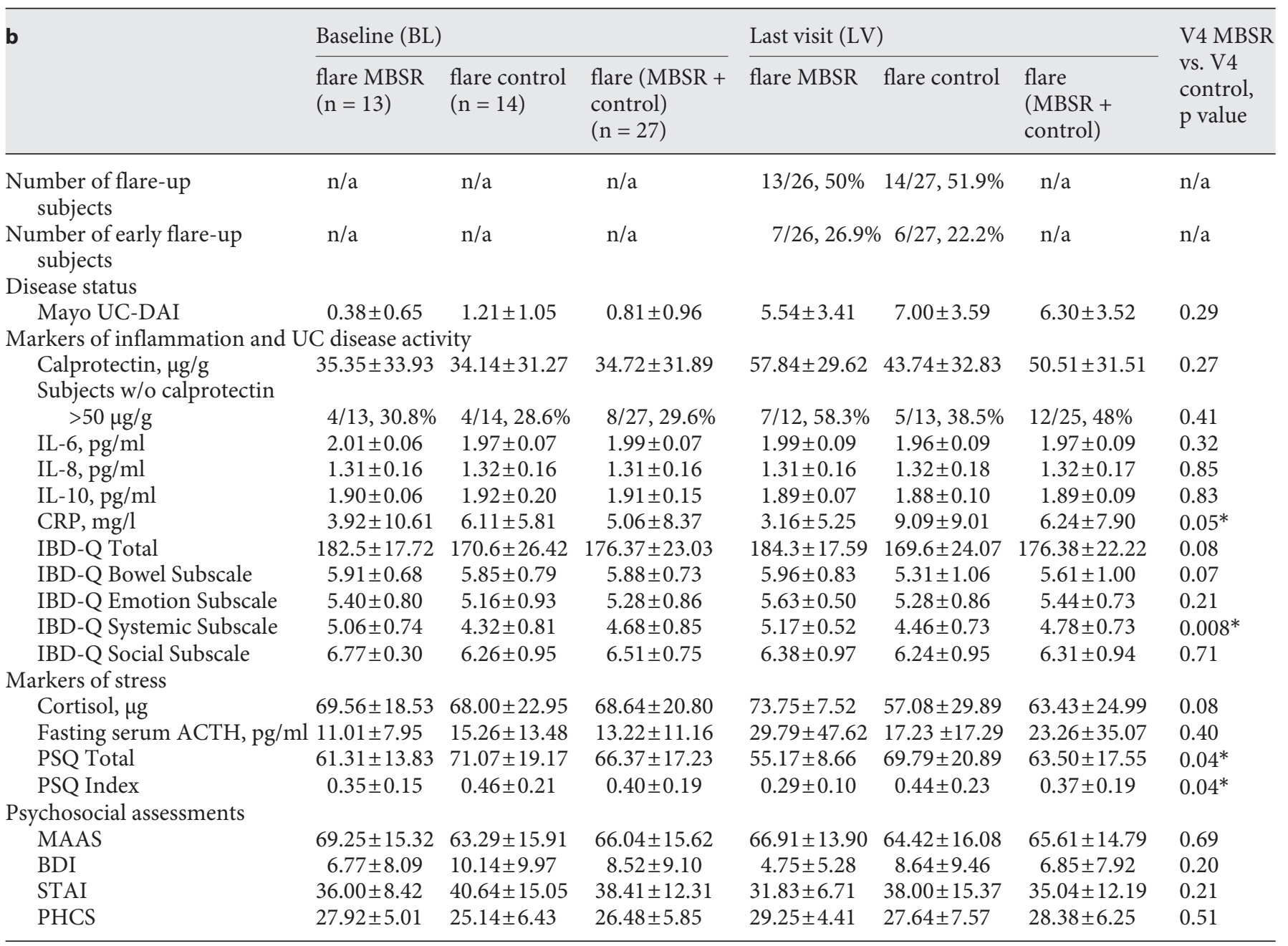

Values expressed as mean \pm SD. ${ }^{*} \mathrm{p}<0.05$. Early flare-up $<3$ months.

(v) Severity of flare: Data from the mixed model analysis (table 3 ) and between group comparisons during the last visit showed no differences in severity of flareup in patients who flared during the 12-month study period (table 4b, Mayo UC-DAI: MBSR $=5.54 \pm 3.41 \mathrm{vs}$. control $=7.0 \pm 3.59$ ).

(b) Markers of stress:

(i) 24-Hour urinary cortisol and serum ACTH: There was a significant difference $(\mathrm{p}=0.007)$ in serum ACTH levels over time between flared subjects in the MBSR group compared to flared subjects in the control group. There were no significant changes over the course of the study in urinary cortisol levels in non-flare and flare-up subjects in either group (table 3 ). However, there was a trend for increasing urinary cortisol at the time of flareup in the MBSR group; while urinary cortisol in the control group tended to decrease at the time of flare-up (table $4 \mathrm{~b})$.

(ii) PSQ scores: The mixed model analysis showed no significant changes over the course of the study in PSQ total and index scores among non-flared and flared-up subjects (table 3 ). However, among patients who flared, those in the MBSR group demonstrated significantly lower PSQ total $(55.17 \pm 8.66)$ and index scores $(0.29 \pm 0.10)$ at last visit, compared to patients who flared in the control group (PSQ total: $69.79 \pm 20.89$; PSQ index: $0.44 \pm 0.23$; $\mathrm{p}=0.04$; table $4 \mathrm{~b})$. 
(c) Psychological assessments:

(i-iv) There were no differences between MBSR subjects who flared and control subjects who flared on measures of depression, anxiety mindfulness, or perceived health competence (BDI: $\mathrm{p}=0.64$; STAI: $\mathrm{p}=0.12$; MAAS: $\mathrm{p}=0.91$; PHCS: $\mathrm{p}=0.30$ ) (table 3 ) nor were there differences between the last-visit scores of the subjects who flared in the MBSR and control groups (BDI: $p=0.20$; STAI: $\mathrm{p}=0.21$; MAAS: $\mathrm{p}=0.69$; PHCS: $\mathrm{p}=0.51)(\mathrm{ta}-$ ble $4 \mathrm{~b})$. Likewise, there were no differences between MBSR and control groups on measures of depression (BDI: $\mathrm{p}=0.75$ ), anxiety (STAI: $\mathrm{p}=0.92$ ), mindfulness (MAAS: $\mathrm{p}=0.43$ ) or perceived health competence (PHCS: $\mathrm{p}=0.33$ ), among non-flared subjects at last visit.

\section{Was There a Subset of Patients Who Responded to MBSR Treatment? Post hoc Analysis}

In order to determine if there was a subset of UC patients who might benefit from MBSR, we performed a post hoc analysis taking baseline characteristics into consideration, as factors that may be contributing significantly to MBSR response. Subjects were split into tertiles (upper, middle and lower). Subjects in the MBSR group with the most severe, gastrointestinal symptoms at baseline (i.e. upper tertile of the IBD-Q Bowel Subscale) demonstrated a positive MBSR effect, compared to those exhibiting less gastrointestinal severe symptoms (upper tertile vs. lower tertile, $\mathrm{p}<0.001$; fig. 3a), an effect that was not observed in the control group. Likewise, MBSR subjects with the highest tertile of PSQ scores at baseline demonstrated a reduced flare-up rate compared to control group subjects also in the highest tertile (fig. 3b). Similar to the PSQ data, flare-up rate among MBSR subjects in the top tertile of cortisol at baseline was reduced, compared to controls with comparable baseline cortical levels (fig. 3c).

\section{Discussion}

To our knowledge, this is the first study to assess the therapeutic efficacy of MBSR on UC patients who were in remission. In contrast to our hypothesis, there was no overall intervention effect on disease outcome or markers of mucosal inflammation (calprotectin) or systemic inflammation (IL-6 and IL-8) and change in CRP was not large enough to be clinically significant to affect UC flareup. Significant differences in both IL-10 and ACTH levels were found when comparing MBSR-flared subjects with

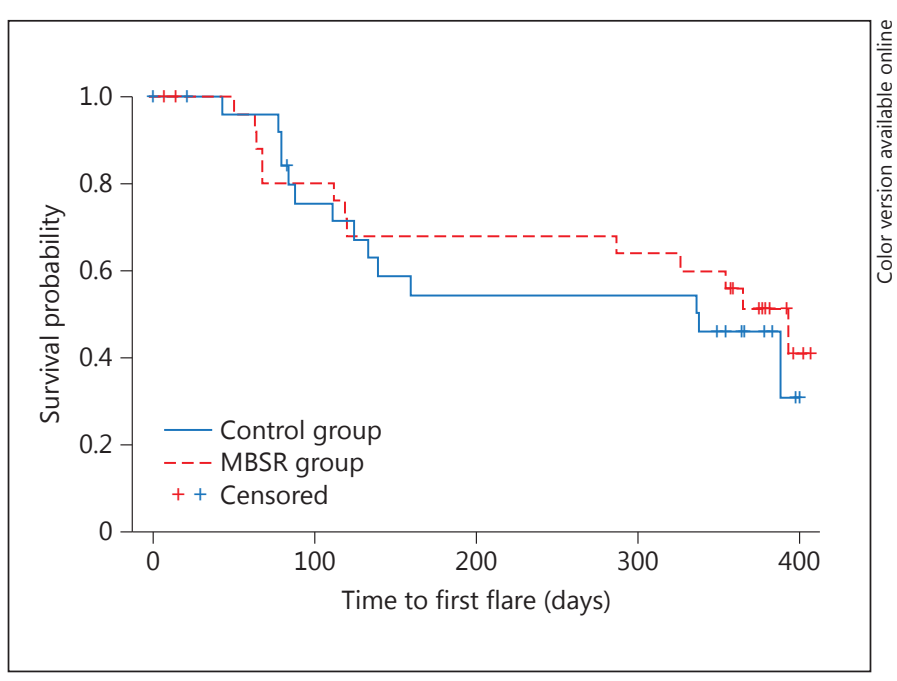

Fig. 2. Kaplan-Meier survival analysis. Comparison of time to flare from baseline to last visit (flare-up). Time to first flare: as measured in number of days from baseline visit to flare-up.

control flares over time. It appears that MBSR had a biological impact on cytokine levels (IL-10) and ACTH, although not enough to prevent flare-up. Further research is necessary to determine how MBSR may impact systemic inflammation and the stress response. MBSR did not affect the proportion of participants who flared, severity of flare-up or time to flare-up in patients with moderately severe UC; however, MBSR had a significant positive impact on the QOL in UC patients who flared (IBD$\mathrm{Q}$ score, $\mathrm{p}<0.01$, table 3 ). Patients in the control group experienced a statistically significant decrease in QOL (i.e. IBD-Q) at the time of flare, in contrast to the MBSR group who maintained a high QOL even during flare.

The inability of MBSR to impact UC disease course in our study is compatible with MBSR not affecting markers of mucosal or systemic inflammation. One possible reason for this finding is that stress and the dysregulated brain-gut axis were not the primary triggers of flare-ups in many of our patients, thus mind/body interventions would not be expected to be effective. Prior research suggests that triggers for IBD flare-ups are diverse and that stress may trigger flare-up in only a subset of patients [5]. Indeed, the use of mind/body interventions to alter disease course for a subset of UC patients is consistent with data from the Cochrane review of psychological interventions for IBD patients, where the authors concluded that further evidence is necessary to assess the efficacy of specific psychological interventions for subsets of patients needing psychological interventions [28]. There- 

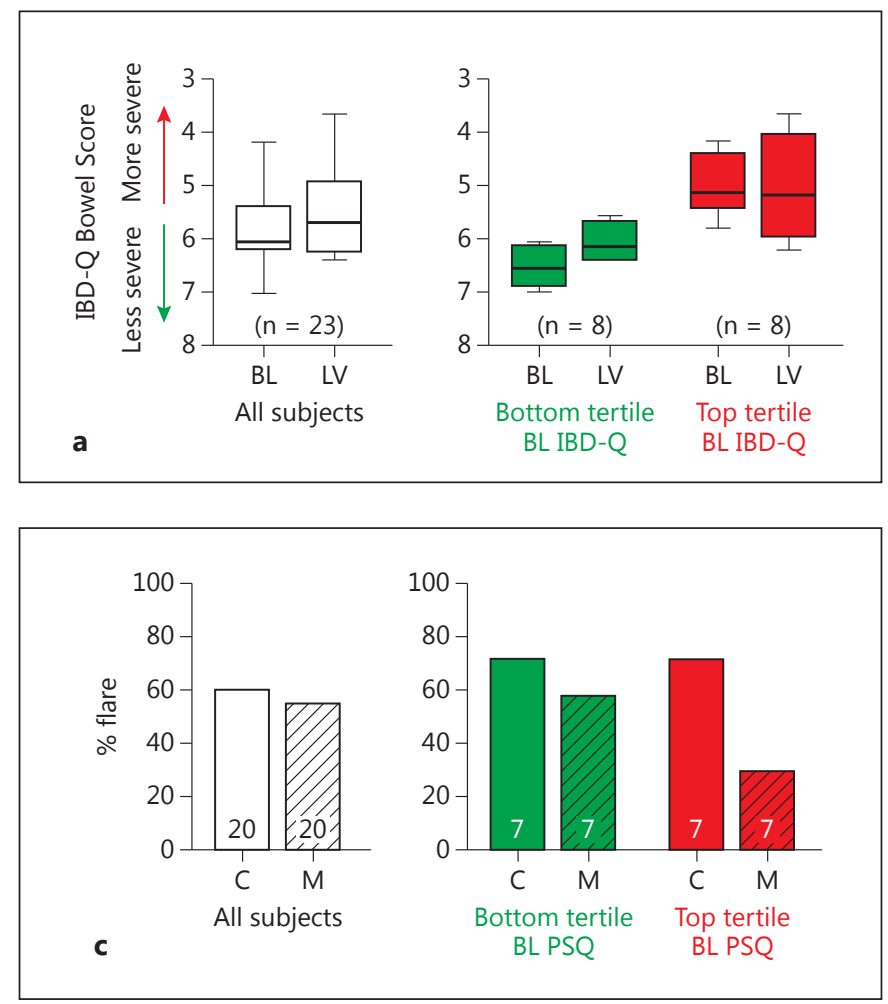

Fig. 3. A subset of UC patients responded to MBSR treatment. a, b IBD-Q Bowel Subscale: when all subjects were pooled together there were no significant differences between self-reported IBDQ bowel symptoms at baseline (BL) or during the last visit (LV) for subjects in the control (a) or MBSR (b) groups (Student's t test: control, $\mathrm{p}=0.262 ; \mathrm{MBSR}, \mathrm{p}=0.509)$. Separating subjects into tertiles based on the baseline IBD-Q scores [i.e. those with the least severe/bottom tertile (6/19), moderately severe/middle tertile (not shown), and most severe/top tertile (6/19)] revealed that patients with the most severe IBD-Q bowel symptoms at baseline responded positively to MBSR (b), an effect that was not observed in the control group (a). Two-way repeated measures ANOVA: control, tertile $\mathrm{F}(1,31)=34.129, \mathrm{p}<0.0001$; visit $\mathrm{F}(1,31)=2.361, \mathrm{p}=0.147$; interaction $\mathrm{F}(1,31)=4.645, \mathrm{p}=0.049$. MBSR, tertile $\mathrm{F}(1,23)=$ $16.456, \mathrm{p}=0.002$; visit $\mathrm{F}(1,23)=18.825, \mathrm{p}=0.002$; interaction

fore, it is reasonable that if mind/body interventions prevent flare-up by minimizing the impact of stress on inflammatory cascades, only individuals with heightened physiological responses to stress would benefit. Our post hoc analysis provides preliminary evidence for this notion: patients with the highest PSQ scores and the highest urinary cortisol concentrations had less frequent flareups with MBSR compared to controls (fig. 3). It should be noted that tailoring treatment based on patient characteristics is a well-accepted approach in IBD management (e.g. pharmacotherapy). The concept of tailoring
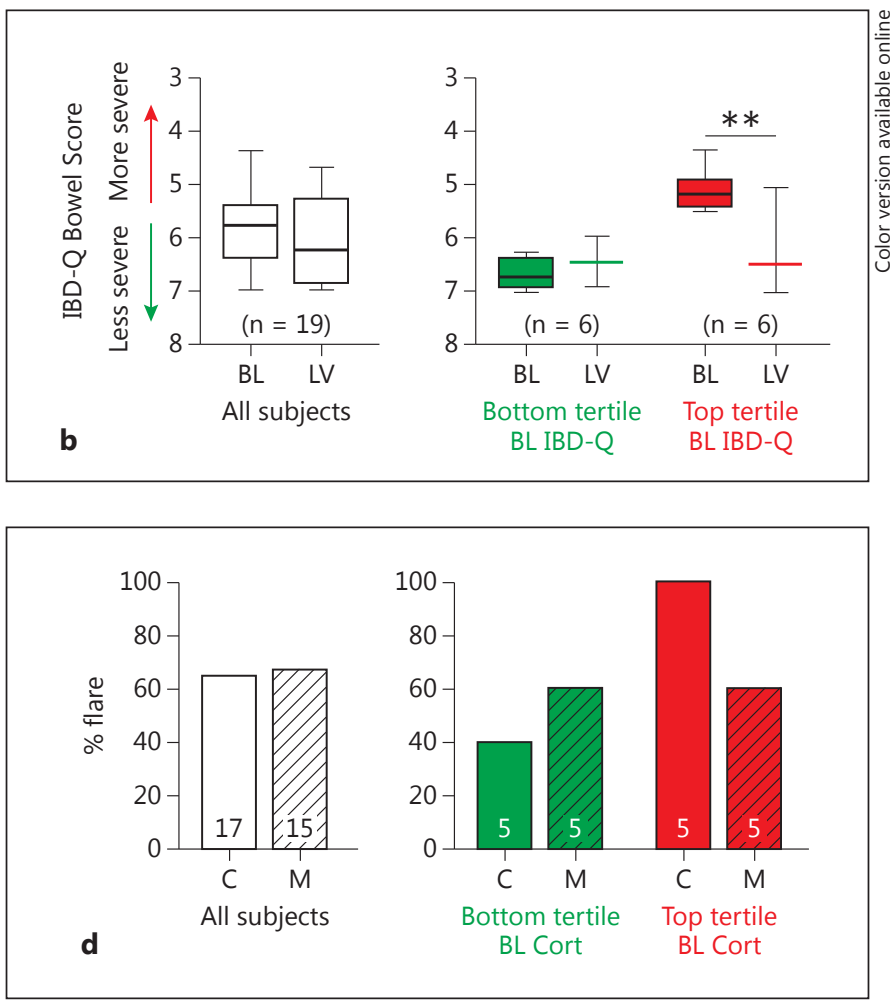

$\mathrm{F}(1,23)=45.813, \mathrm{p}<0.0001 .{ }^{* *}$ Post hoc Tukey significance, $\mathrm{p}<$ 0.01. c Perceived Stress Questionnaire (PSQ): when all subjects were pooled together, the percent of subjects that flared between control (C) and MBSR (M) subjects was similar. Separating subjects into tertiles based on baseline PSQ scores suggests that those subjects with the highest perceived stress at baseline may have a reduction in flare, an effect that was not observed in the control group. d Urinary cortisol (Cort): when all subjects were pooled together, the percent of subjects that flared between control (C) and MBSR (M) subjects was similar. Separating subjects into tertiles based on baseline urinary cortisol suggests that those subjects with the physiological stress response at baseline may have a reduction in flare, an effect that was not observed in the control group.

intervention options has also been suggested in the MBSR literature [29]. Another possible reason for failure of MBSR to impact UC disease course is choice of the study cohort. Further studies are needed to determine whether mind/body interventions could be effective in inducing remission in patients with active UC. Lack of response to MBSR does not necessarily mean that other mind/body interventions, or more intensive MBSR interventions, are ineffective for altering disease course. For example, longer, more sustained interventions might be needed for people with more frequent exacerbations. 
Moreover, we believe that our study cohort is the one who is most in need of additional treatment. That is, those with mild disease are already doing well on current treatment and those with severe UC require rather urgent interventions (like surgery or inpatient treatment), and an intervention like MBSR would not be appropriate. Thus, our findings are generalizable for those with moderate to moderately severe UC.

Patients in the current study reported a high QOL at the time of enrollment (IBD-Q > 170) and QOL remained excellent in those who did not flare-up regardless of intervention assignment, likely reflecting a QOL ceiling effect which could not be improved upon. Among subjects who flared, the IBD-Q scores dropped in subjects in the control group, an effect that was prevented in subjects who received MBSR ( $<0.001$, table 3$)$. Similarly, patients who flared in the MBSR group reported less flareinduced stress assessed with the PSQ (baseline 61 vs. flare-up 55) while in the control group patients PSQ score remained stable (baseline 71.1 vs. flare-up 69.8) $(\mathrm{p}=0.04)$. Thus, MBSR administered during UC remission seems to have altered both perceived stress and QOL during flare-ups. This outcome is compatible with the findings of several prior studies of other mind/body interventions in patients with inactive IBD. For example, a recent randomized trial of 114 IBD patients, where adding stress management psychotherapy (i.e. cognitive behavioral therapy and psychoeducation) to treatment as usual, had no overall effect on disease course, but improved disease-specific QOL over treatment as usual [30]. Additionally, a review by von Wietersheim and Kessler [31] concluded that a range of psychotherapies (i.e. stress management; supportive psychotherapy and behavior therapy) did not have a positive impact on disease course, but in some instances, positively influenced psychological function.

In our study, MBSR decreased perceived stress during flare-ups but did not impact other factors of psychological state. This suggests that during flare-up, the impact of MBSR training of participants to face physical symptoms and subsequent emotional responses was, at least partially, distinct from previous reports demonstrating MBSR-induced amelioration of depression and anxiety [32]. The positive impact of MBSR on QOL and perceived stress, during flare among chronically ill patients, has not been previously reported and is consistent with MBSR teaching the skill of facing physical distress with mental ease.

In our study, MBSR did not significantly affect anxiety or depression in UC subjects and this differs from prior studies where MBSR impacted depression and anxiety. Our results may be due to a ceiling effect resulting from the self-reported good mental health at baseline in our cohort. Similarly, the relatively high state of mindful attention and awareness (i.e. MAAS) at the time of enrollment likely caused a ceiling effect that precluded finding any treatment-induced improvements in MAAS. This may explain how patients with such high scores had dramatic improvement in QOL and perceived stress, without changes in mindfulness. Alternately, it is possible that the MAAS, which focuses primarily on observing, and less on the other four facets of mindfulness (describing, acting with awareness, non-judging of inner experience, and non-reactivity to inner experience) [33] may have limited our ability to detect a change in mindfulness. These findings suggest that baseline mindfulness using the MAAS, or other measures, might be an important sampling criterion for future studies of MBSR, especially in populations with good mental health.

The observed trend in reduced urinary cortisol suggests that MBSR may decrease brain-gut axis hyperactivity as well as the psychological responses to stress (as measured by the PSQ), but markers of inflammation were not impacted. It could be that MBSR does not influence inflammation sufficiently to alter disease course or that MBSR practice did not begin soon enough to develop enough skillfulness to stop progress in pre-flare biologic processes. Urinary cortisol was increased in MBSR subjects who flared (69.6-73.8), a finding that was not observed in the control group (68.0-57.1) (table $4 \mathrm{~b}, \mathrm{p}=$ 0.08 ). This finding may indicate that a rise in cortisol may be a 'useful' and physiologically beneficial biological response, but that the increase in cortisol may not have been sufficient to prevent flare-up, but may have contributed to the improvement in QOL.

There are some study limitations to consider. Our study included a relatively small sample size, thereby limiting our ability to consider potential impacts of diseaseassociated variables, such as medications on outcomes. Secondly, the small sample size may have resulted in a type 2 error and thus we failed to show significant effects of MBSR on disease outcome. If the result of our post hoc analysis is confirmed in a future study, the effect size of an MBSR intervention for a subset of UC patients is substantial and clinically relevant. Therefore, in spite of our small sample size, we believe our conclusion is clinically relevant and accurate. That is, an MBSR intervention to prevent UC flare-up may not effective for all UC patients, but it might be useful in a subset of UC patients with a high state of stress and an exaggerated response to stress. 
Of course, our study showed that MBSR could still be a useful adjuvant therapy to improve QOL for UC patients, even if it does not impact disease course. Third, subjects who flared provided physiological and psychological data at the time of flare-up, which constituted their final visit, while those who did not flare were examined at fixed intervals. Thus, the potential effects of active disease on these variables might have masked the intervention's effects. Fourth, participants in the MBSR group were significantly older both at the time of diagnosis as well as at the time of study participation compared to those in the control group, however it is unlikely that these age differences had any impact on the results since there is no evidence that age impacts UC disease course in adults. Moreover, there were no statistically significant differences between groups on any of the baseline disease characteristics. Finally, it is unfortunate we could not have both a wait list control and a psychoeducational control; it is possible both the MBSR group and control group affected depression and anxiety, and the inclusion of these additional groups would have provided insight.

In summary, our study demonstrated that a non-medical, 8-week time-intensive mind/body intervention was well tolerated by, and highly acceptable to, IBD patients. We found that the 8-week course of MBSR did not significantly affect markers of inflammation and did not affect time, severity, or proportion of participants who flared in patients with moderately severe UC in remission. Despite this finding, MBSR prevented a flare-induced drop in QOL compared to controls. Thus, it is reasonable to consider incorporating mind/body interventions in the management of UC as it is well tolerated and well accepted and appears to minimize the negative impact of flare-up on QOL. Furthermore, our post hoc analysis suggests that MBSR might be effective in decreasing the rate of flare-ups in patients with heightened state of stress. Further studies using a larger cohort are required to substantiate our post hoc findings.

\section{Acknowledgements}

This study was supported by a grant from the National Complementary Center for Alternative Medicine of the National Institutes of Health (Grant No. 5R21AT003939-02) We would like to thank the study subjects who participated in this study for their time and dedication; members of the data safety and monitoring board: Janine Gauthier, PhD, Sohrab Mobarhan, MD, Kimberly Skarupski, $\mathrm{PhD}$, Vered Hankin, $\mathrm{PhD}$, for teaching several of the MBSR courses; Jeff Dusek, MD, for his advice in developing the curriculum for the time/attention control group; Eimile Dalton, BA, Mary Marshall, BA, Delia Daian, MD, and Nazia Kazmi, MS, for their assistance in recruiting subjects and tracking all visits, and Rachel Goldsmith, $\mathrm{PhD}$, for her thoughts on the manuscript. The study was registered with clinicaltrail.govandhad theidentifier,NCT00568256.

\section{References}

1 Keighly MRB, Stockbrugger RW: Inflammatory bowel disease. Aliment Pharm Ther 2003;18(suppl 3):66-70.

$\checkmark 2$ Navarro F, Hanauer S: Treatment of inflammatory bowel disease: safety and tolerability issues. Am J Gastroenterol 2003;98:S18-S23.

3 Kane SV, Cohen R, Aikens JE, et al: Predictors of non-compliance with mesalamine in quiescent ulcerative colitis. Am J Gastroenterol 2001;96:2929-2932.

4 Levenstein S, Prantera C, Varvo V, et al: Stress and exacerbation in ulcerative colitis: a prospective study of patients enrolled in remission. Am J Gastroenterol 2000;95:1213-1219.

5 Maunder RG, Levenstein S: The role of stress in the development and clinical course of inflammatory bowel disease: epidemiological evidence. Curr Mol Med 2008;8:247-252.

6 Farhadi A, Keshavarzian A, Van de Kar LD, et al: Heightened responses to stressors in patients with inflammatory bowel disease. Am J Gastroenterol 2005;100:1796-1804.

7 Mawdsley JE, Macey MG, Feakins RM, et al: The effect of acute psychologic stress on systemic and rectal mucosal measures of inflam- mation in ulcerative colitis. Gastroenterology 2006;131:410-419.

$>8$ Kabat-Zinn J: Mindfulness-based interventions in context: past, present, and future. Clin Psychol Sci Pract 2003;10:144-156.

-9 Chiesa A, Serretti A: Mindfulness-based stress reduction for stress management in healthy people: a review and meta-analysis. J Altern Complement Med 2009;15:593-600.

10 Speca M, Carlson LE, Goodey E, et al: A randomized, wait-list controlled clinical trial: the effect of a mindfulness meditation-based stress reduction program on mood and symptoms of stress in cancer outpatients. Psychosom Med 2000;62:613-622.

11 Gross CR, Kreitzer MJ, Russas V, et al: Mindfulness meditation to reduce symptoms after organ transplant: a pilot study. Adv Mind Body Med 2004;20:20-29.

12 Grossman P, Tiefenthaler-Gilmer U, Raysz A, Kesper U: Mindfulness training as an intervention for fibromyalgia: evidence of postintervention and 3-year follow-up benefits in well-being. Psychother Psychosom 2007;76: 226-233.
13 Rosenzweig S, Greeson JM, Reibel DK, et al: Mindfulness-based stress reduction for chronic pain conditions: variation in treatment outcomes and role of home meditation practice. J Psychosom Res 2010;68:29-36.

14 Carlson LE, Speca M, Patel KD, et al: Mindfulness-based stress reduction in relation to quality of life, mood, symptoms of stress, and immune parameters in breast and prostate cancer. Psychosom Med 2003;65:571-578.

15 Robinson FP, Mathews HL, Witek-Janusek L: Psycho-endocrine-immune response to mindfulness-based stress reduction in individuals infected with the human immunodeficiency virus: a quasiexperimental study. J Altern Complement Med 2003;9:683-694.

16 Gaylord SA, Palsson OS, Garland EL, et al: Mindfulness training reduces the severity of irritable bowel syndrome in women: results of a randomized controlled trial. Am J Gastroenterol 2011;106:1678-1688.

17 Jedel S, Merriman P, Hoffman A, et al: Relationship of mindfulness, quality of life and psychiatric symptoms among patients with ulcerative colitis. Mindfulness 2013;4:296-300. 
18 Kabat-Zinn J: Full Catastrophe Living: Using the Wisdom of Your Body and Mind to Face Stress, Pain and Illness. New York, Delacorte Press, 1990

19 Schroeder KW, Tremaine WJ, Ilstrup DM: Coated oral 5-aminosalcylic acid therapy for mildly to moderately active ulcerative colitis. N Engl J Med 1987;317:1625-1629.

20 Irvine EJ: Quality of life issues in patients with inflammatory bowel disease. Am J Gastroenterol 1997;92:18S-24S.

21 Levenstein S, Prantera C, Varvo V, et al: Development of the perceived stress questionnaire: a new tool for psychosomatic research. J Psychosom Res 1993;37:19-32.

22 Beck AT: Depression: Causes and Treatment. Philadelphia, University of Pennsylvania Press, 1970, p 193.

23 Carlson JF, Waller NG: Review of the Beck Depression Inventory; in Impara JC, Plake BS, Murray LL (eds): Thirteenth Mental Measurement Yearbook. Lincoln, Boros Institute of Mental Measurement/University of Nebraska, 1998, pp 100-121.
24 Spielberger CD, Sydeman SJ: State-trait anxiety inventory and state-trait anger inventory; in Maruish M (ed): The Use of Psychological Testing for Treatment Planning and Outcome Assessment. Hillsdale, Erlbaum, 1974, pp 292-321.

25 Carlson LE, Brown KW: Validation of the mindful attention awareness scale in a cancer population. J Psychosom Res 2005;58:29-33.

26 Bishop SR: What do we really know about mindfulness-based stress reduction? Psychosom Med 2002;64:71-84.

27 Smith M, Wallston KA, Smith C: The development and validation of the perceived health competence scale. Health Educ Res 1995;10: 51-64.

28 Timmer A, Preiss JC, Motschall E, et al: The Cochrane Collaboration Psychological interventions for treatment of inflammatory bowel disease. Cochrane Database Syst Rev 2011; 2:CD006913.
29 Grossman P, Niemann L, Schmidt S, Walach $\mathrm{H}$ : Mindfulness-based stress reduction and health benefits. A meta-analysis. J Psychosom Res 2004;57:35-43.

30 Boye B, Lundin KE, Jantschek G, et al INSPIRE study: does stress management improve the course of inflammatory bowel disease and disease-specific quality of life in distressed patients with ulcerative colitis or Crohn's disease? A randomized controlled trial. Inflamm Bowel Dis 2011;17:1863-1873.

-31 Von Wietersheim J, Kessler H: Psychotherapy with chronic inflammatory bowel disease patients: a review. Inflamm Bowel Dis 2006;12: 1175-1184

32 Grootenhuis MA, Maurice-Stam H, Derkx BH, Last BF: Evaluation of a psychoeducational intervention for adolescents with inflammatory bowel disease. Eur J Gastroenterol Hepatol 2009;21:430-435.

33 Baer RA, Smith GT, Hopkins J, et al: Using self-report assessment methods to explore facets of mindfulness. Assessment 2006;13: $27-45$. 